\title{
Odd Ramanujan Sums of Complex Roots of Unity
}

\author{
Soo-Chang Pei, Fellow, IEEE, and Kuo-Wei Chang
}

\begin{abstract}
A special class of odd-symmetric length-4N periodic signals is studied, and it is shown how the odd Ramanujan sums are used as weighting coefficients to compute their pure imaginary discrete Fourier transform (DFT) integer-valued coefficients. The odd Ramanujan sum, being the sums of complex roots of unity, can be calculated either using closed-form formulas or computed recursively through the impulse response of a derived infinite impulse response (IIR) filter. This special class of odd-symmetric signals and odd Ramanujan sums can be combined together with the previous even-symmetric special class signals and the well-known even Ramanujan sums as a useful tool for signal processing.
\end{abstract}

Index Terms-Complex roots of unity, discrete Fourier transform (DFT), infinite impulse response (IIR) filter, Ramanujan sums.

\section{INTRODUCTION}

I N 1950, Cohen investigated a special class of even-symmetric periodic signals, the so-called even arithmetical functions [1], [2]. The discrete Fourier transform (DFT) coefficients [3] of this class of signals can be computed by forming a weighted average of the signals, using integer-valued coefficients. These integer-valued weighting coefficients are special sums of complex roots of unity, the well-known even Ramanujan sums [4], [5], which can be computed using closed-form formulas. Recently, a recursive method was introduced to compute the even Ramanujan sums through a derived infinite impulse response (IIR) filter [6].

The main objective of this letter is the introduction and study of another special class of odd-symmetric periodic signals $\mathrm{x}_{r}(n)$ for which odd Ramanujan sums of the complex roots of unity can be efficiently combined to compute the DFT coefficients. The signals are defined based on the number of theoretic properties of the time index $n$ and the period $r$. We show that the DFT coefficients of this class of signal can be computed by forming a weighted average using imaginary integer-valued coefficients rather than the usual imaginary real-valued trigonometric functions.

We are interested in the odd signal by noting that only the even arithmetical function $\mathrm{c}_{r}(n)$ is discussed in [6]. However, in fact, this kind of even function can be transformed into an odd function by simple circular shifts, under a special condition. Thus, we can use the methods like [6] to build up the co-

Manuscript received October 20, 2005; revised May 14, 2006. This work was supported by the National Science Council, R.O.C., under Contracts NSC 94-2213-E-002-072 and NSC 93-2752-E-002-006-PAE. The associate editor coordinating the review of this manuscript and approving it for publication was Dr. Xiang-Gen Xia.

The authors are with the Department of Electrical Engineering, National Taiwan University, Taipei 10617, Taiwan, R.O.C. (e-mail: pei@cc.ee.ntu.edu. tw).

Digital Object Identifier 10.1109/LSP.2006.881527 efficients and employ the cyclotomic polynomials to introduce an IIR system.

The motivation to study the odd signals is to enhance the method in [6]. Observing that, in [6], the maximum possible number of distinct values that an even signal $(\bmod r)$ can have is $\tau(r)=\prod_{p^{\text {prime }}}\left(\mathrm{m}_{\mathrm{p}}+1\right)$ if $r=\prod_{p^{\text {prime }}} p^{m_{p}}$. So if $r=12$, then we can only use six distinct values. However, if we combine the even signals with the odd ones, the values we can use are expanding to eight, as we will see later. Since there is an odd part, we must deal with the imaginary integer-valued coefficients and the real one, which becomes a Gaussian integer, while in [6], only real integer-valued coefficients are considered.

The structure of this letter is as follows. First, in Section II, we define a class of odd-symmetric periodic signals and show how the odd Ramanujan sums are used as weighting coefficients to compute their Fourier coefficients. Then we combine the odd part with the even part. Circular shift is also introduced, and we prove that our method is better than that, theoretically and practically. A z-domain approach to analyze the signals is presented in Section III. The theoretical development is supplemented by concrete examples throughout this letter. Conclusions are drawn in Section IV.

\section{Odd Signals $(\bmod r)$ AND TheIR FouRIER COEFFICIENTS}

In this section, we define and analyze a class of discrete-time periodic signals, which henceforth will be called odd signals $(\bmod r)$. The signals are defined with respect to a fixed positive integer $r$ for all integer values of the time index $n$. Let $\operatorname{gcd}(a, b)$ denote the greatest common divisor of the integer $a$ and $b$. A signal $\mathrm{x}_{r}(n)$ is called an odd signal $(\bmod r)$ if

$$
x_{r}(n)=\left\{\begin{array}{ll}
x_{r}(\operatorname{gcd}(n, r)), & \text { if } \frac{r}{\operatorname{gcd}(n, r)} \equiv 0 \bmod 4, \\
-x_{r}(\operatorname{gcd}(n, r)), & \text { and } \frac{r}{\operatorname{gcd}(n, r)} \equiv 1 \bmod 4 \\
& \text { and } \frac{n}{\operatorname{gcd}(n, r)} \equiv 0 \bmod 4, \quad \forall n . \\
0, & \text { elsewhere }
\end{array} \quad \forall n .\right.
$$

From the above definition, it follows that $\mathrm{x}_{\mathrm{r}}(n)$ is a signal $(\bmod r)$ is periodic with period $r$, i.e., $\mathrm{x}_{\mathrm{r}}(k r+n)=\mathrm{x}_{\mathrm{r}}(n)$. In addition, $\mathrm{x}_{\mathrm{r}}(\mathrm{n})$ processes odd symmetry, in other words, $\mathrm{x}_{r}(r-$ $n)=-\mathrm{x}_{r}(n)$. The proof is in the Appendix.

As an example of a signal satisfying (1), let $r=12$. (Note that any nonzero signal should satisfy $r \equiv 0 \bmod 4$.) The value of the signal $\mathrm{x}_{12}(n)$ in its main period may be generally represented by a sequence of numbers of the form

$$
\begin{aligned}
& \left\langle x_{12}(n), n=0 \ldots 11\right\rangle \\
& \quad=\langle 0, p, 0, q, 0, p, 0,-p, 0,-q, 0,-p\rangle .
\end{aligned}
$$

We observe that despite the limitation imposed by the definition, the signal is still rich in content and can assume distinct values in its main period. We are, thus, interested in determining 
the maximum possible number of distinct values that $\mathrm{x}_{r}(n)$ can assume for a given $r$. By the method similar to [6], we can get that, in general, for a given $r=4 \prod_{p \text { prime }} p^{m_{p}}$, there are $\tau(r)=\prod_{p \text { prime }}\left(\mathrm{m}_{\mathrm{p}}+1\right)$ different values that $x_{r}(n)$ can assume.

In addition, we can use the same idea to rewrite

$$
x_{r}(n)=\sum_{\substack{d \mid r \\ d \geq 1}} x_{r}\left(\frac{r}{d}\right) h_{r, d}(n)
$$

where the signal $h_{r, d}(n)$ is periodic with period $r$, which is defined for $n \in[0, r-1]$ as

$$
\begin{aligned}
& h_{r, d}(n) \\
& \quad= \begin{cases}1, & \text { if } \frac{r}{\operatorname{gcd}(n, r)}=d \equiv 0 \bmod 4, \frac{d n}{r} \equiv 1 \bmod 4 \\
-1, & \text { if } \frac{r}{\operatorname{gcd}(n, r)}=d \equiv 0 \bmod 4, \frac{d n}{r} \equiv 3 \bmod 4 \\
0, & \text { otherwise. }\end{cases}
\end{aligned}
$$

It is clear that the signals $h_{r, d}(n)$ are odd $(\bmod r)$.

Let $i=\sqrt{ }-1$ and use $1=i^{4 k},-1=i^{4 k+2}, \forall k \in N$, so

$$
h_{r, d}(n)= \begin{cases}i \cdot i^{-\frac{d n}{r}}, & \text { if } \frac{r}{\operatorname{gcd}(n, r)}=d \equiv 0 \bmod 4 \\ 0, & \text { otherwise. }\end{cases}
$$

So the DFT of $h_{r, d}(n)$ is computed using a sum of the form

$$
H_{r, d}(n)=\sum_{0 \leq k \leq r-1} h_{r, d}(k) W_{r}^{-n k}
$$

where $W_{r}=\exp (2 \pi i / r)$. Using (5), we can rewrite (6) as

$$
\begin{aligned}
H_{r, d}(n) & =\sum_{0 \leq k \leq r-1} h_{r, d}(k) W_{r}^{-n k} \\
& =i \cdot \sum_{\substack{0 \leq k \leq r-1 \\
0 \leq k^{\prime} \leq d-1 \\
\text { gcd }\left(d, k^{\prime}\right)=1 \\
d=0 \text { mod } 4}} W_{r}^{-n k} \cdot i^{-\frac{d k}{r}} \\
& =i \cdot W^{-n k^{\prime}} \cdot i^{-k^{\prime}}
\end{aligned}
$$

where $k^{\prime}=d k / r$, and then using $i=W_{d}^{d / 4}$, so

$$
\begin{aligned}
H_{r, d}(n) & =i \cdot \sum_{\substack{0 \leq k^{\prime} \leq d-1 \\
\operatorname{gcd}\left(d, k^{\prime}\right)=1 \\
d \equiv 0 \bmod 4}} W_{d}^{-n k^{\prime}} \cdot i^{-k^{\prime}} \\
& =i \cdot \sum_{\substack{0 \leq k^{\prime} \leq d-1 \\
\operatorname{gcd}\left(d, k^{\prime}\right)=1 \\
d \equiv 0 \bmod 4}} W_{d}^{-k^{\prime}\left(n+\frac{d}{4}\right)} .
\end{aligned}
$$

The last complex sum in (8) is another odd type of the Ramanujan sums [2]. We use the symbol $\mathrm{c}_{d}(n)$ to denote the value of the even Ramanujan sum $\sum_{\substack{0 \leq U \leq d-1 \\ \operatorname{gcd}(U, d)=1}} W_{d}^{-n U}$ and write

$$
H_{r, d}(n)=i \cdot c_{d}\left(n+\frac{d}{4}\right), \quad d \geqslant 1, \quad d \equiv 0 \bmod 4
$$

It can be shown that $c_{d}(n)$ is an integer, so it will be $c_{d}(n+$ $d / 4)$. It is important to note that although $c_{d}(n)$ is an even func- tion, $\mathrm{c}_{d}(n+d / 4)$ is an odd function of $n$ if $\mathrm{d} \equiv 0 \bmod 4$. To show this, let $y=n+d / 4$, so $\mathrm{n}-\mathrm{d} / 4=\mathrm{y}-\mathrm{d} / 2$, and

$$
\begin{aligned}
& c_{d}\left(-n+\frac{d}{4}\right)=c_{d}\left(n-\frac{d}{4}\right)=c_{d}\left(y-\frac{d}{2}\right) \\
& =\sum_{\substack{0 \leq U \leq d-1 \\
\operatorname{gcd}(U, d)=1}} W_{d}^{-\left(y-\frac{d}{2}\right) U} \\
& =\sum_{\substack{0 \leq U \leq d-1 \\
\operatorname{gcd}(U, d)=1}} \exp \left(\frac{-i 2 \pi \cdot\left(y-\frac{d}{2}\right) U}{d}\right) \\
& =\sum_{\substack{0 \leq U \leq d-1 \\
\operatorname{gcd}(U, d)=1}} \exp \left(\frac{-i 2 \pi y}{d}+i \pi U\right) \\
& =\sum_{\substack{0 \leq U \leq d-1 \\
\operatorname{gcd}(U, d)=1}} \exp \left(\frac{-i 2 \pi y}{d}\right) \exp (i \pi U) \\
& \because \operatorname{gcd}(U, d)=1 \quad \text { and } \quad d \equiv 0 \bmod 4 \\
& \Rightarrow U \equiv 1 \bmod 2 \Rightarrow \exp (i \pi U)=-1 \\
& \therefore c_{d}\left(-n+\frac{d}{4}\right) \\
& =\sum_{\substack{0 \leq U \leq d-1 \\
\operatorname{gcd}(U, d)=1}} \exp \left(\frac{-i 2 \pi y}{d}\right) \exp (i \pi U) \\
& =-\sum_{\substack{0 \leq U \leq d-1 \\
\operatorname{gcd}(U, d)=1}} \exp \left(\frac{-i 2 \pi y}{d}\right) \\
& =-c_{d}(y)=-c_{d}\left(n+\frac{d}{4}\right) \text {. }
\end{aligned}
$$

The proof is complete.

To compute the DFT coefficients $\mathrm{X}_{r}(n)$ of the odd signal $\mathrm{x}_{r}(n)$, we invoke the linearity of the DFT operation. Using (3), (4), (6), and (9), we can write

$$
X_{r}(n)=i \sum_{\substack{d \mid r \\ d>1 \\ d \equiv 0 \bmod 4}} x_{r}\left(\frac{r}{d}\right) c_{d}\left(n+\frac{d}{4}\right) .
$$

It can be verified that the DFT coefficients $\mathrm{X}_{r}(n)$ are also odd. Thus, we only need to compute $\tau(r)$ different values for $\mathrm{X}_{r}(n)$. These values are

$$
\begin{aligned}
& X_{r}\left(\frac{r}{D}\right)=i \sum_{\substack{d \mid r \\
d>1 \\
d \equiv 0 \bmod 4}} x_{r}\left(\frac{r}{d}\right) c_{d}\left(\frac{r}{D}+\frac{d}{4}\right), \\
& D>1, \quad D \mid r
\end{aligned}
$$

and $X_{r}(0)=0$.

As a concrete example, let us compute the $\tau(12)=2$ distinct Fourier coefficients for $x_{12}(n)$. Using (11), we have

$$
\begin{aligned}
X_{12}(0) & =0=X_{12}(2)=X_{12}(4)=X_{12}(6) \\
& =X_{12}(8)=X_{12}(10) \\
X_{12}(1) & =X_{12}(5)=-X_{12}(7)=-X_{12}(11) \\
& =-2 i x_{12}(1)-2 i x_{12}(3) \\
X_{12}(3) & =-X_{12}(9)=-4 i x_{12}(1)+2 i x_{12}(3) .
\end{aligned}
$$


To inversely compute the signal values from their Fourier coefficients $\mathrm{X}_{r}(n)$, we can use the same decomposition approach as (3) and note that the complex conjugate of the sum given by (9) is $-i c_{d}(n+d / 4)$. More precisely, the sequence $\mathrm{x}_{r}(n)$ is the sum for the inverse DFT

$$
x_{r}(n)=\frac{1}{r} \sum_{k=0}^{r-1} X_{r}(n) W_{r}^{n k}
$$

It is decomposable by using $h_{r, d}(n)$, where $d$ is a positive divisor of $r$, and therefore, (11) is simplified as

$$
x_{r}(n)=\frac{-i}{r} \sum_{\substack{d \mid r \\ d>1 \\ d \equiv 0 \bmod 4}} X_{r}\left(\frac{r}{d}\right) c_{d}\left(n+\frac{d}{4}\right) .
$$

Our next step is to study when we put together the even signals and odd signals $(\bmod r)$. Assume $r=12$; then from [6], the values of the even signal in its main period may be represented by a sequence of numbers of this form

$$
\left\langle y_{12}(n), n=0 \ldots 11\right\rangle=\langle a, b, c, d, e, b, f, b, e, d, c, b\rangle
$$

and from (2)

$$
\begin{aligned}
\left\langle x_{12}(n), n=0\right. & \ldots 11\rangle \\
& =\langle 0, p, 0, q, 0, p, 0,-p, 0,-q, 0,-p\rangle .
\end{aligned}
$$

Combining (15-1) and (15-2), we can get

$$
\begin{aligned}
& \left\langle z_{12}(n), n=0 \ldots 11\right\rangle \\
& \quad=\langle a, p+b, c, q+d, e, p+b, f, b \\
& \quad-p, e, d-q, c, b-p\rangle .
\end{aligned}
$$

As we can see, the values we can use are: $a, p+b, c, q+$ $d, e, f, b-p, d-q$, eight distinct values in total. Compared with (15-1), which can only use six values, we find that (15-3) has $33 \%$ increase. As a matter of fact, when $r$ is big, the values, or what we called dimensions, have nearly $100 \%$ increase from that in [6]. We use an example to demonstrate that. If $r=2^{n}$, then, from [6], the dimensions are $n+1$, but the odd part provides another $n-1$ dimensions. So overall, it has $2 n$ dimensions, which have increased by $100 \%$ when $n \gg 2$.

Before we go to the next section, let us see another way to increase dimensions from [6]. From the circular shift property of DFT [3, (8.87), p. 567], we know that if

$$
x[n] \stackrel{\mathrm{DFT}}{\longleftrightarrow} X[k] \quad 0 \leq n \leq r-1
$$

then

$$
\begin{aligned}
& x[(n-m) \bmod r], \\
& \qquad 0 \leq n \leq r-1 \stackrel{\mathrm{DFT}}{\longleftrightarrow} e^{-j(2 \pi k / r) m} X[k] .
\end{aligned}
$$

This property can be used for the even signals $(\bmod r)$, especially for $r \equiv 0 \bmod 4$. If $m=r / 4$, the right side of (16) becomes $e^{-i(2 \pi k / 4)} X[k]=(-i)^{k} X[k]$, and thus, if the coefficients of $X[k]$ are integer-valued, then $(-i)^{k} X[k]$ is also "integer-valued," with some imaginary parts. We can use (15-1) as our example. Because $r=12$ and so we choose $m=3$ and use (16), we can get

$$
\begin{aligned}
\left\langle y_{12}^{\prime}(n), n=0 \ldots 11\right\rangle & \\
& =\left\langle d^{\prime}, c^{\prime}, b^{\prime}, a^{\prime}, b^{\prime}, c^{\prime}, d^{\prime}, e^{\prime}, b^{\prime}, f^{\prime}, b^{\prime}, e^{\prime}\right\rangle .
\end{aligned}
$$

One can easily verify that DFT $\left(\mathrm{y}_{12}^{\prime}(n)\right)$ is complex-integer weighted sum of $\mathrm{y}_{12}^{\prime}(n)$. More importantly, we combine (17) with (15-1)

$$
\begin{aligned}
& \left\langle x_{12}(n), n=0 \ldots 11\right\rangle \\
& =\left\langle d^{\prime}+a, c^{\prime}+b, b^{\prime}+c, a^{\prime}+d, b^{\prime}+e, c^{\prime}\right. \\
& \left.\quad+b, d^{\prime}+f, e^{\prime}+b, b^{\prime}+e, f^{\prime}+d, b^{\prime}+c, e^{\prime}+b\right\rangle
\end{aligned}
$$

and notice that generally, $d^{\prime}+a \neq a^{\prime}+d$ so the signal above has eight dimensions, namely, $d^{\prime}+a, c^{\prime}+b, b^{\prime}+c, a^{\prime}+d, b^{\prime}+$ $e, d^{\prime}+f, e^{\prime}+b, f^{\prime}+d$, which is at the same position with (15-3). However, we should be careful that this new method is not always the same as the old ones. Taking $r=8$ as a counter example, our odd part method provides six dimensions

$$
\begin{aligned}
& \left\langle x_{8}(n), n=0 \ldots 7\right\rangle \\
& \quad=\left\langle a, b+b^{\prime}, c+c^{\prime}, b+b^{\prime}, d, b-b^{\prime}, c-c^{\prime}, b-b^{\prime}\right\rangle
\end{aligned}
$$

while the circular shift method only provides five

$$
\begin{aligned}
& \left\langle x_{8}^{\prime}(n), n=0 \ldots 7\right\rangle \\
& =\left\langle a+c^{\prime}, b+b^{\prime}, c+a^{\prime}, b+b^{\prime}, d\right. \\
& \left.\quad+c^{\prime}, b+b^{\prime}, c+d^{\prime}, b+b^{\prime}\right\rangle .
\end{aligned}
$$

This counter example leads us to the theorem.

Theorem: dimensions of even-odd method $\geqslant$ dimensions of circular shift method.

Proof: From (16), we know that if $\operatorname{gcd}\left(r, k_{1}\right)=$ $\operatorname{gcd}\left(r, k_{2}\right)$, and $k_{1} \neq k_{2} \bmod 4$, then $k_{1}$ and $k_{2}$ will "build up" different values while using the circular shift method and, thus, increase the dimensions.

However, from (1), we found that using even-odd method, the condition becomes

$$
\begin{aligned}
& \operatorname{gcd}\left(r, k_{1}\right)=\operatorname{gcd}\left(r, k_{2}\right), \quad \text { and } \\
& \frac{k_{1}}{\operatorname{gcd}\left(r, k_{1}\right)} \neq \frac{k_{2}}{\operatorname{gcd}\left(r, k_{2}\right)} \bmod 4 .
\end{aligned}
$$

So the circular method is just a special case of the even-odd method, with $\operatorname{gcd}\left(r, k_{1}\right)=\operatorname{gcd}\left(r, k_{2}\right)=1$, which implies that the even-odd method might have more $\left(k_{1}, k_{2}\right)$ pairs (in other words, dimensions of even-odd method $\geqslant$ dimensions of circular shift method). The proof is complete.

In practice, one may find that it is easier to decompose the original signal into the even-odd signal than to decompose it by the circular shift method. The reason is that we are more familiar with the first one, by using

$$
\frac{x_{r}(n)+x_{r}(-n)}{2} \text { and } \frac{x_{r}(n)-x_{r}(-n)}{2},
$$

where $\mathrm{x}_{r}(n)$ is the original signal. 


$$
\begin{aligned}
& x_{r}(r-n)= \begin{cases}x_{r}(\operatorname{gcd}(r-n, r)), & \text { if } \frac{r}{\operatorname{gcd}(r-n, r)} \equiv 0 \bmod 4, \\
-x_{r}(\operatorname{gcd}(r-n, r)), & \text { if } \frac{r}{\operatorname{gcd}(r-n, r)} \equiv 1 \bmod 4 \\
& \text { and } \frac{r-n}{\operatorname{gcd}(r-n, r)} \equiv 0 \bmod 4, \\
0, & \text { elsewhere }\end{cases} \\
& \begin{cases}x_{r}(\operatorname{gcd}(r-n, r)), & \text { if } \frac{r}{\operatorname{gcd}(r-n, r)} \equiv 0 \bmod 4, \\
& \text { and } \frac{n}{\operatorname{gcd}(r-n, r)} \equiv 3 \bmod 4\end{cases} \\
& = \begin{cases}-x_{r}(\operatorname{gcd}(r-n, r)), & \text { if } \frac{\operatorname{gcd}(r-n, r)}{\operatorname{gcd}(r-n, r)} \equiv 0 \bmod 4, \\
& \text { and } \frac{n}{\operatorname{gcd}(r-n, r)} \equiv 1 \bmod 4 \\
0, & \text { elsewhere }\end{cases} \\
& \text { elsewhere }
\end{aligned}
$$

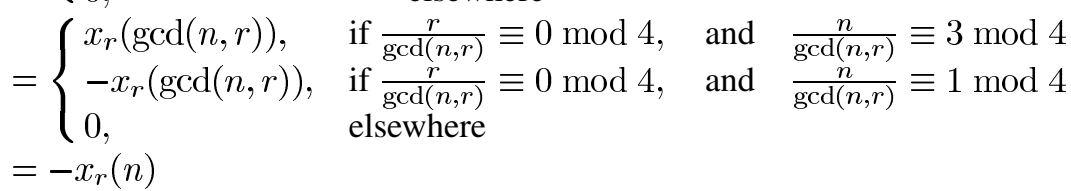

\section{Z-DOMAIN CHARACTERIZATION OF ODD SIGNALS AND ODD RAMANUJAN SUMS}

The purpose of this section is to develop a z-domain representation of the odd signals $\bmod (r)$. From (14), the one-sided $\mathrm{z}$-transform of $x_{r}(n)$ is of the form

$$
\sum_{n=0}^{\infty} x_{r}(n) z^{-n}=\frac{-i}{r} \sum_{\substack{d \mid r \\ d>1 \\ d \equiv 0 \bmod 4}} X_{r}\left(\frac{r}{d}\right) C_{d}^{\prime}(z)
$$

where $\mathrm{C}_{\mathrm{d}}^{\prime}(z)$ is the one-sided z-transform of the odd Ramanujan sums $\mathrm{c}_{d}(n+d / 4)$. Using the concept of shift property of one sided z-transform, we can obtain

$$
C_{d}^{\prime}(z)=z^{\frac{d}{4}} C_{d}(z)-\sum_{i=1}^{\frac{d}{4}} z^{i} c_{d}\left(\frac{d}{4}-i\right)
$$

where $\mathrm{C}_{\mathrm{d}}(z)$ is given by [6]. As an example, the closed-form expressions for $\mathrm{C}_{12}^{\prime}(z)$ and $\mathrm{C}_{12}(z)$ are given by

$$
C_{12}^{\prime}(z)=\frac{-2 z^{-1}-2 z^{-3}}{1-z^{-2}+z^{-4}}
$$

and

$$
C_{12}(z)=\frac{4-2 z^{-1}}{1-z^{-2}+z^{-4}}
$$

\section{CONCLUSION}

In this letter, we have shown that a special class of odd periodic signals $(\bmod r)$ possess a DFT representation, with the imaginary integer-valued odd Ramanujan sums playing the role of the complex-valued roots of unity. In addition, combining this odd part with the even part [6], one can get more dimensions. We have also derived an IIR system suitable for these odd signals.

\section{APPENDIX}

Proof of odd symmetry property of $\mathrm{x}_{\mathrm{r}}(\mathrm{n})$ :

We can use the facts that $\operatorname{gcd}(a, b)=\operatorname{gcd}(a \bmod b)$ and $\operatorname{gcd}(0, b)=b$ and then rewrite (1) as shown in the equation at the top of the page.

\section{REFERENCES}

[1] E. Cohen, "A class of arithmetical functions," Proc. Nat. Acad. Sci. USA, vol. 41, pp. 939-944, 1955.

[2] — "Representations of even functions $(\bmod r)$. I. Arithmetical identities," Duke Math. J., vol. 25, pp. 401-421, 1958.

[3] A. V. Oppenheim, R. W. Schafer, and J. R. Buck, Discrete-Time Signal Processing, 2nd ed. Englewood Cliffs, NJ: Prentice-Hall, 1989.

[4] S. Ramanujan, "On certain trigonometrical sums and their application in the theory of numbers," Trans. Comb. Phil. Soc., vol. 22, pp. 259-276, 1918

[5] M. Planat, H. Rosu, and S. Perrine, "Ramanujan sums for signal processing of low-frequency noise," Phys. Rev. E, vol. 66, p. 51128, 2002.

[6] S. Samadi, M. O. Ahmad, and M. N. S. Swamy, "Ramanujan sums and discrete Fourier transforms," IEEE Signal Process. Lett., vol. 12, no. 4, pp. 293-296, Apr. 2005. 